\title{
Characterization and performance improvement of the large titanium sublimation pumps in the AUG and W7-X neutral beam injectors
}

\author{
G. Orozco, M. Froeschle, B. Heinemann, C. Hopf, R. Nocentini, R. Riedl \\ Max Planck Institute for Plasma Physics, EURATOM Association, Boltzmannstr. 2, 85748 Garching, Germany
}

The experimental devices ASDEX Upgrade (AUG) and Wendelstein 7-X (W7-X) are equipped with two neutral beam injectors each for plasma heating with up to $20 \mathrm{MW}$. Four large titanium sublimation pumps $(\mathrm{TSPs})\left(4 \times 1.5 \times 0.2 \mathrm{~m}^{3}\right)$ in each injector provide proper vacuum conditions (below $10^{-2} \mathrm{~Pa}$ ) during the $10 \mathrm{~s}$ beam pulse with a gas feed of up to $30 \mathrm{~Pa} \times \mathrm{m}^{3} / \mathrm{s}$. A maximum pumping speed of up to $3000 \mathrm{~m}^{3} / \mathrm{s}$ for $\mathrm{H}_{2}$ is obtained by frequent renewal of the Ti coating at the pump surfaces. Ti is sublimed from $4 \mathrm{~m}$ long ohmically heated hanging pairs of Ti sublimation wires. So far, all pairs of wires were operated with constant current during $120 \mathrm{~s}$, despite of the large spread of the resistance of the wires. Tests have been performed in a dedicated test facility in order to characterize the pumping performance for $\mathrm{H}_{2}$ with different parameters for sublimation power and time. The data gathered can be used to simulate fully operative TSP systems for real NBI operation scenarios in AUG and W7-X, so as to find an optimal sublimation strategy for the TSPs.

Keywords: Wendelstein 7-X; ASDEX-Upgrade; Neutral Beam Injection; Vacuum technology; Titanium sublimation pump

\section{Introduction}

In the last three years, annual campaigns have been performed to test and upgrade the large IPP titanium sublimation pumps (TSPs) of the neutral beam injection system (NBI) for AUG, to the operation conditions in its replica NBI system of W7-X. The TSPs are operated with DC in between plasma discharges of AUG, when the magnetic field coils are off. The superconducting coils of W7-X permanently create a stray magnetic field in the torus hall, incompatible with the DC operation of the TSPs. In the first experimental campaign, the operation with AC was proved to be safe and reliable within equivalently strong magnetic fields as of in the W7-X torus hall, and as result a standard heating pulse with sine waveform and ramped frequency from 200 to $500 \mathrm{~Hz}$ was selected [1].

The second campaign aimed at increasing the availability of the TSP and the operation time at full TSP performance. The operation campaigns at $\mathrm{W} 7-\mathrm{X}$ are expected to be longer than in AUG, with more frequent NBI pulses. In AUG the performance of the TSPs may decrease at the end of long campaigns. Over time, the hanging wires where $\mathrm{Ti}$ is sublimed from (sublimators) start failing, typically through electric contacts. At this stage, the Ti reservoir in the sublimators is still large but not usable anymore. The main cause is the accumulative lengthening due to material creep of the sublimator core. A modified Ti sublimator with a different supporting core has been tested and validated [2]. The original pure Tantalum (Ta) core is replaced with a Ta-W alloy (2.5\%), which does not significantly impact the electrical properties of the sublimators but reduces the lengthening over a factor of 20 .

The third campaign focused on improving the pumping performance of the TSP regarding the sublimation parameters in order to make the best use of its Ti reservoir. First, the main parameters involved during sublimation of the TSP are identified. Second, an evaluation method is described in order to characterize the vacuum system, obtaining the pumping speed and its relation with the pressure and the saturation level of the Ti deposited layers.

\section{Sublimation of Ti for gas gettering}

Fresh deposited metallic Ti reacts with the striking gas molecules to build semi-stable compounds (chemisorption). The adsorption or "getter" effect depends primarily on the properties of the deposited $\mathrm{Ti}$ layer, the gas kind and its thermodynamic state $(\mathrm{p}, \mathrm{T})$ and geometric factors that define the flux of particles hitting an active Ti layer, mainly the geometry of the pump. From now on, all experiences named in this paper will refer only to molecular hydrogen being fed at room temperature and in the defined geometry of the IPP TSP within the NBI injector standard vacuum vessel [3].

The properties of the deposited Ti layer include its temperature the layer thickness and its roughness. The weak covalent chemical bounds are sensitive to temperature change and may release the gas molecules again (outgassing). The thickness and roughness of the Ti layer depend again on sublimation rate of Ti from the Ti source, the sublimation time and the geometry of the pump, relative position and distance of the deposited surfaces to the Ti source. While some authors point out an optimum thickness [4], others describe the need of large roughness, which is achieved through higher deposition rates [5].

\subsection{Sublimation rate control}

The Ti sublimator is electrically heated, ideally, below the melting temperature of $\mathrm{Ti}$ in order to increase the sublimation rate. So far, the TSPs are operated with a predefined current for all pairs of sublimators. This 
current starts at $139 \mathrm{~A}$ and it is incremented over time by a few A, when the operator observes a poor pumping performance of the TSP, seldom over $145 \mathrm{~A}$. This approach neglects the big spread in the electric resistance of the sublimators, which changes along their operation life [2]. The spread is as large as $\pm 15 \%$ at an early use stage of the wires which translates into a $\pm 15 \%$ spread in the heating power, typically from 28 to $36 \mathrm{~kW}$. Within this power range, a simple radiation model of the sublimators (Figure 1) estimates that the temperature of the wire would lay within a $100 \mathrm{~K}$ interval. The sublimation rate is estimated to differ around a factor of five. This effect can be observed in the vessel pressure evolution during the Ti sublimation in one TSP (Figure 2). Within the first $30 \mathrm{~s}$, the pressure rises strongly, due to the quickly heating up and outgassing of the sublimators themselves (phase a). Simultaneously $\mathrm{Ti}$ deposits onto the walls and starts gettering gas so the pressure returns back to the start value and even further down (phase b). The higher the heating power, the lower the pressure burst reaches and the sooner it gets back to initial pressure.

An intuitive conclusion is that a good control of the electric power could lead to a more accurate control of the temperature and thus of the sublimation rate. This work will investigate the effect on pumping performance for electrical power in the described range $28-36 \mathrm{~kW}$ with $2 \mathrm{~kW}$ increments.

\subsection{Optimum sublimation time}

The sublimation time determines as well the thickness of the deposited Ti layers. At IPP the pulse duration traditionally lasts $120 \mathrm{~s}$, based on experience and in order to avoid the outgassing of the walls. These are water cooled but have a rather limited cooling capacity, so the temperature rises during the sublimation and the walls may start outgassing. After the pressure has returned to the start value, it stays stable for a while within the phase c, but increases again slowly till the sublimation cycle ends (phase d).

The outgassing of the walls should be avoided, so for each sublimation power, its optimum sublimation time is defined as the time at the end of its phase $\mathrm{c}$. The

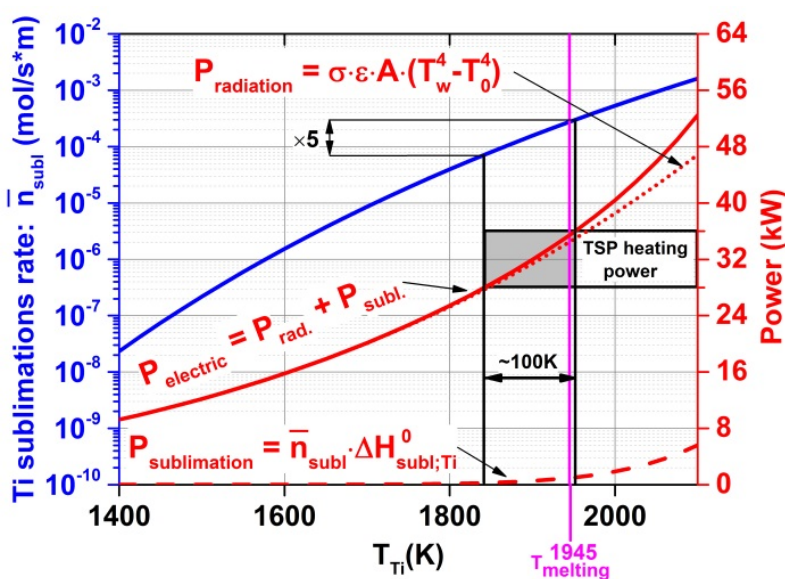

Figure 1: Ti sublimation rate density per Ti sublimator length unit as a function the Ti temperature [6] (left). Sublimation power (mostly radiated) as a function of the temperature of the Ti sublimator (right).

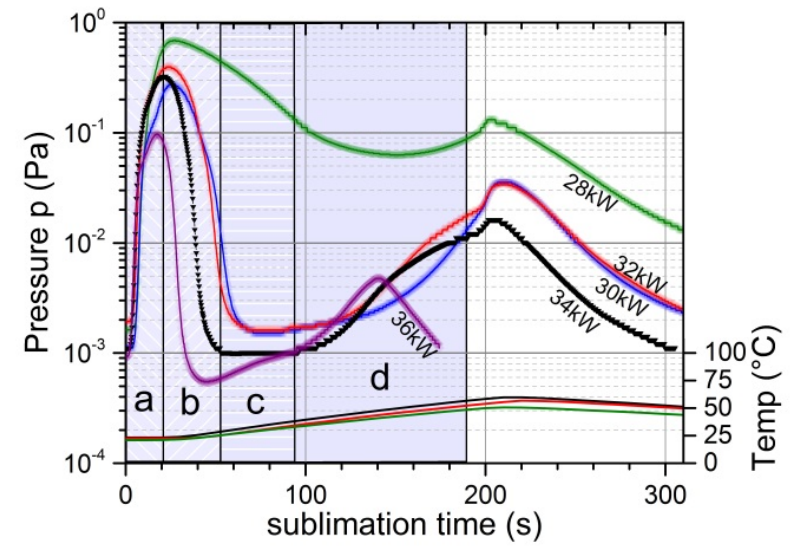

Figure 2: Vessel pressure evolution during sublimation in one TSP chamber for different heating powers during $180 \mathrm{~s}$. Four phases can be recognized $a, b, c, d$. Measured temperature of the walls through a thermocouple (bottom).

impact on the pump performance of the reference sublimation time of $120 \mathrm{~s}$ and the optimum sublimation time will be compared within this work.

\section{Characterization of the NBI vacuum system}

The NBI vacuum vessel has a volume of $\sim 30 \mathrm{~m}^{3}$ fully-equipped. It has several tenths of meters of O-Ring seals with vacuum to air and vacuum to water boundaries. The base vacuum pumping system consists of two parallel branches with a turbopump $(2.2 \mathrm{~m} / \mathrm{s}$, $10^{-2} \mathrm{~Pa}$ ) and a forepump each. They slowly pump down after vessel venting to $10^{-3}-10^{-4} \mathrm{~Pa}$, depending on the inherent presence of air leaks. The four IPP large TSP are required to keep pressure under $10^{-2} \mathrm{~Pa}$ during the standard $10 \mathrm{~s}$ neutral beam pulse, when up to $30 \mathrm{~Pa} \cdot \mathrm{m}^{3} / \mathrm{s}$ of $\mathrm{H}_{2}$ is fed into the NBI vessel. The pumping speed required for all four TSPs together is $3000 \mathrm{~m}^{3} / \mathrm{s}$. The TSPs rely on two factors to achieve this high pumping speed. First, a large Ti deposition surface $\left(\sim 45 \mathrm{~m}^{2}\right.$ per TSP), achieved thanks to its large dimensions $\left(4 \times 1.5 \times 0.3 \mathrm{~m}^{3}\right)$, its chambered configuration and the special corrugation of the deposition surfaces. Second, the active getter surfaces are embedded directly at the sides of the vacuum vessel so that the conductance loss is negligible.

In getter pumps however, the pumping speed degrades with the saturation of the gettering surfaces. This is quantified with the surface coverage $(\theta)$ or the number of molecules adsorbed per surface unit $\left(\mathrm{m}^{-2}\right)$. A new Ti layer is deposited (chamber re-activation), when the pumping performance is too low. In a typical operation day of AUG all chambers are sublimed with fresh $\mathrm{Ti}$ once before the first AUG discharge. Two chambers per TSP are usually re-activated during the 20 minutes pause between the NBI pulses. This kind of operation scheme leads to an unsymmetrical pumping characteristics of the chambers, so a precise estimation of the pumping performance is difficult.

In this investigation only one chamber will be activated. In order to describe the behavior of more active chambers together, a simplified simulation is proposed in the end. 


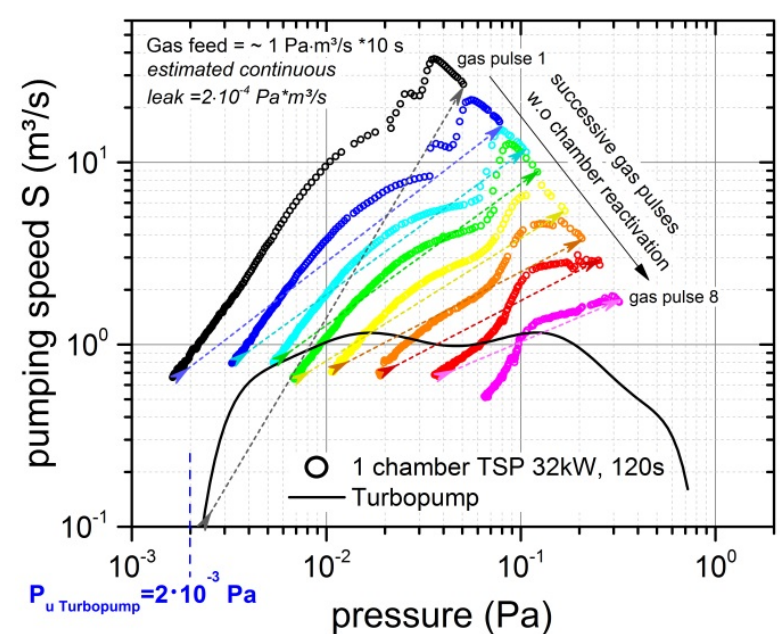

Figure 3: Pumping speed to vessel pressure for a once activated TSP chamber $(32 \mathrm{~kW}, 120 \mathrm{~s})$ for eight successive $1 \mathrm{~Pa} \cdot \mathrm{m}^{3} / \mathrm{s}$ gas puffs. The pumping speed of the turbopump line is shown for comparison.

\subsection{Test stand for TSP characterization}

The vacuum vessel is identical to the NBI injectors of AUG and W7-X. It contains just a TSP, a turbopump line via a gate valve that can separate it from the vacuum vessel, the standard gas system of an injector and two pressure gauges (cold cathode - IKR251 from Pfeiffer). Both pressure measurements are averaged and fast enough in the pressure range investigated (over $10^{-4} \mathrm{~Pa}$ ) with a response time of less than $10 \mathrm{~ms}$ for a sample rate of $0.1 \mathrm{~s}$.

The turbopump line evacuates the vessel down to around $10^{-3} \mathrm{~Pa}$, which is the ultimate pressure achievable without any Ti sublimation. By shutting the gate valve, a constant air leak of $2 \cdot 10^{-4} \mathrm{~Pa} \cdot \mathrm{m}^{3} / \mathrm{s}$ can be calculated from the rate of pressure increase, that is around ten times higher than the best values observed at the full-equipped NBI injectors but still negligible compared to the injected $\mathrm{H}_{2}$ gas feed $\left(\dot{q}_{H_{2}}\right)$ of $1 \mathrm{~Pa} \cdot \mathrm{m}^{3} / \mathrm{s}$. This has been previously calibrated and corresponds roughly to the nominal operation gas feed in AUG $\left(30 \mathrm{~Pa} \cdot \mathrm{m}^{3} / \mathrm{s}\right)$ divided by the number of total sublimation chambers (36). The duration of the gas puff is always $10 \mathrm{~s}$.

For the characterization of the single TSP chamber, the gate valve shuts before the sublimation pulse starts. A pair of short-lived sublimators $(3 \mathrm{~h}$ of use from theoretical $16 \mathrm{~h}$ possible) is then activated for the selected sublimation powers and sublimation times. As soon as the pressure turns back to the start pressure, the first gas puff is injected. Successive gas puffs follow without any $\mathrm{Ti}$ sublimation in between, once the pumping speed goes below $1 \mathrm{~m}^{3} / \mathrm{s}$. After several gas puffs, the maximum pumping speed reached is comparable to that of the turbopump (Figure 3) and the experiment concludes.

\subsection{Pumping speed of the TSP}

The instantaneous pumping speed is calculated from the vacuum pressure evolution and the known gas feed (1).

$$
s(t)=-(\underbrace{\frac{\dot{q}_{H_{2}}(t)}{p(t)}}_{\text {gas feed }}-\underbrace{\frac{d p(t)}{d t} \cdot \frac{V}{p(t)}}_{\text {pressure change }})\left(\frac{m^{3}}{s}\right)
$$

In Figure 3, the pump characterization is shown for the reference TSP sublimation parameters $(32 \mathrm{~kW}$, $120 \mathrm{~s}$ ). From the start with $\sim 10^{-3} \mathrm{~Pa}$ and negligible pumping speed, the maximum pumping speed $\left(\sim 40 \mathrm{~m}^{3} / \mathrm{s}\right)$ is obtained within the $10 \mathrm{~s}$ of the first gas puff reaching a pressure of $7 \cdot 10^{-2} \mathrm{~Pa}$. With every successive gas puff, the maximum pumping speed decreases and consequently the pressure peaks at always higher values. The pumping performance degrades very quickly. At a given pressure the pumping speed decreases over four times from puff to puff, as the Ti layer gets filled.

\subsection{TSP pumping speed as a function of the} pressure and the surface coverage

The degradation of the pumping speed can be understood better if the surface coverage is included in the equation. The surface coverage is calculated with the mass balance in the vacuum system. The surface of each TSP chamber is $5 \mathrm{~m}^{2}$ (2).

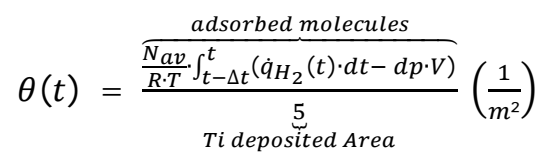

A three dimensional representation of the measured data reveals a convex 3D space, that can be fitted with a $\left(5^{\text {th }}\right.$ degree) polynomial surface $\left(\mathrm{R}^{2}>0.9\right)$ for each of the study cases (Figure 4). This surface allows interpolation for different gas feed scenarios (longer duration, larger gas feeds) and extrapolation towards regions where the experiment does not reach (i.e. $\mathrm{p}<10^{-3} \mathrm{~Pa}, \theta<20 \mathrm{~m}^{-2}$ ). Moreover, it also allows obtaining the pumping speed curve at a given pressure $\left(10^{-2} \mathrm{~Pa}\right.$, in our case $)$, as it is measured in the traditional pumping speed test, by means of a fine controlling of the gas feed that keeps a constant pressure. This is obtained for each of the selected study cases so as to better compare the results (Figure 5).

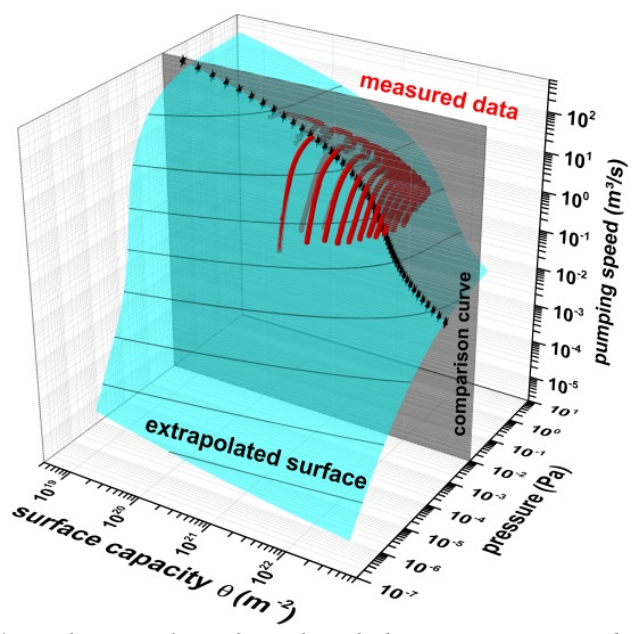

Figure 4: Polynomial surface fit of the pumping speed as a function of the pressure and surface coverage from experimental data. The curve $s=f\left(10^{-2} \mathrm{~Pa}, \theta\right)$ is calculated to compare it among the different experiment cases. 


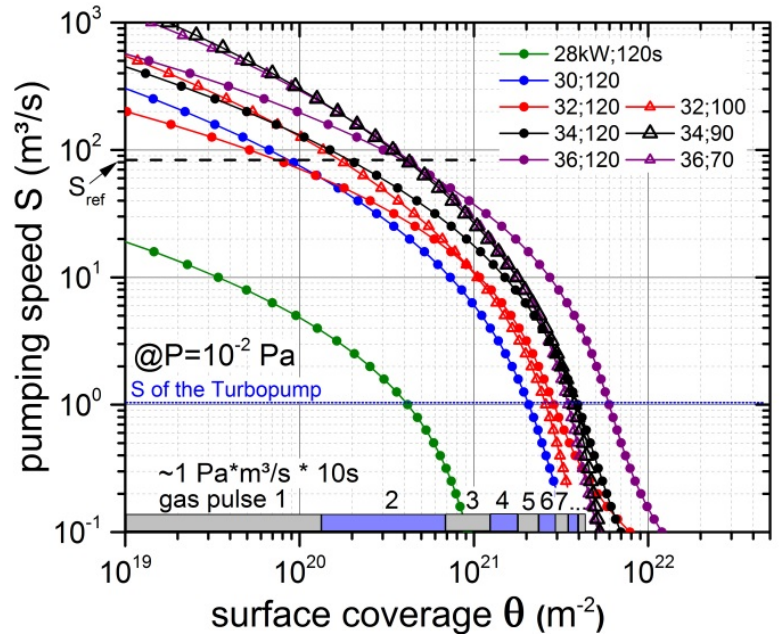

Figure 5: Pumping speed to surface coverage for constant pressure $10^{-2} \mathrm{~Pa}$, for the different sublimation parameters. The sorption coverage reached after each gas puff is showed at the bottom

\section{Results: Sublimation power and time scans}

As expected, the higher the sublimation power, the higher is the pumping speed obtained. After the first gas puff the Ti surface coverage is of around $10^{20}$ molecules $/ \mathrm{m}^{2}$. At the reference pressure, the obtained pumping speed suits the reference value for a single chamber $\left(70 \mathrm{~m}^{3} / \mathrm{s}\right)$ with reference sublimation parameters $(32 \mathrm{~kW}, 120 \mathrm{~s})$. For higher sublimation power, the obtained pumping speed outperforms the reference value even by as much as a factor of four after one gas puff. The best performances are reached however for shorter sublimation times than the referenced $120 \mathrm{~s}$, as it was suspected, depending on the sublimation power. The optimum sublimation parameters are $34 \mathrm{~kW}$ for $90 \mathrm{~s}$, preferred to the very similar case with $36 \mathrm{~kW}$ during $70 \mathrm{~s}$, since the risk of overheating of the wires is less. The degradation of the pumping speed as the Ti surface gets covered is very similar in both cases and keep a higher pumping speed than the reference almost during the two first gas puffs.

\subsection{Multi-chamber simulation}

In real operation as many as 36 chambers are in operation and recursively re-activated. The total pumping speed is the sum of the contributed "s" of each single chamber. A simple 1D simulation on the basis of the experimental data is calculated for all 36 chambers with ideal AUG boundary conditions $\left(30 \mathrm{~Pa} \cdot \mathrm{m}^{3} / \mathrm{s}\right.$ for $10 \mathrm{~s}$ every 20 minutes). The sublimation parameters

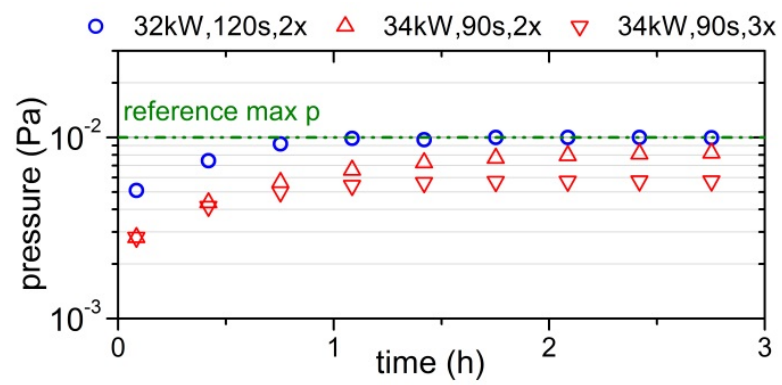

Figure 6: Simulation of a real AUG operation day with all $9 \times 4$ chambers active, for the reference case and the optimized operation $(34 \mathrm{~kW}, 90 \mathrm{~s})$ with two and three sublimation cycles after every AUG discharge. correspond to the reference case $(32 \mathrm{~kW}, 120 \mathrm{~s}$ two times between the AUG discharges) and the optimum case $(34 \mathrm{~kW}, 90 \mathrm{~s}$ with two and three chamber re-activation between discharges). The results in Figure 6 show for the reference case a maximum pressure after three beam pulses around the reference maximum pressure $\left(10^{-2} \mathrm{~Pa}\right)$. The optimum case with three re-activations stays however under almost half the reference maximum pressure.

\section{Conclusions and future work}

Two crucial parameters for the performance of the large IPP TSP have been identified, the sublimation power and the sublimation time. The performance of the pump has been characterized for a single TSP chamber and a set of sublimation powers and times. The best performance is achieved for a sublimation power of $34 \mathrm{~kW}$ during $90 \mathrm{~s}$. It is suggested changing the actual operation schema of constant sublimation current control to a power controlled one. The positive impact of this method on a NBI injector has been simulated but it should be benchmarked in real NBI operation. It can then potentially be used to explore advanced NBI scenarios with different pumping system demands such as longer beam pulses.

There are still some aspects to be addressed in the near future about the impact in the pump efficiency with the aging of the sublimators and the characterization for other relevant gases in NBI systems such as $\mathrm{D}_{2}$ (expected to be similar to $\mathrm{H}_{2}$ ), $\mathrm{N}_{2}, \mathrm{O}_{2}, \mathrm{H}_{2} \mathrm{O}$, etc.

\section{Acknowledgments}

This work has been carried out within the framework of the EUROfusion Consortium and has received funding from the Euratom research and training programme 2014-2018 under grant agreement No 633053. The opinions expressed herein do not necessarily reflect those of Fusion for Energy or the European Commission.

\section{References}

[1] G. Orozco, et al., "AC operation of large titanium sublimation pumps in a magnetic field: results of the test stand for the W7-X neutral beam injectors," Fusion Eng. Des., vol. 89, no. 6-8, pp. 1034-1037, 2014.

[2] G. Orozco, et al., „Optimization of the large titanium sublimation pumps for the neutral beam injection system on AUG and W7-X," IEEE Transactions on plasma science, Bd. PP, Nr. 9, pp. 1-6, 2016.

[3] J.H. Feist, et al., „Large scale titanium getter pumps for the ASDEX-Upgrade neutral beam injectors," in 17th SOFT Fusion Technology, Rome, 1992.

[4] L. Elsworth, L. Holland und L. Laurenson, „The sorption of $\mathrm{N}_{2}, \mathrm{H}_{2}$ and $\mathrm{D}_{2}$ on titanium films at $20^{\circ} \mathrm{C}$ and $-190^{\circ} \mathrm{C}$, “ Harwell internal report number 6a, Crawley, Sussex, 1964.

[5] G.I. Grigorov, „Apparent and real values of common gas sticking coefficients on titanium films and application to getter pump device with periodic active film renovation,“ Vacuum, Bd. 34, pp. 513-517, 1982.

[6] J. M. J. Blocher und I. Campbell, „Vapor pressure of titanium,“ Battelle Memorial Institute, 1949. 